\title{
A Fixed Point Theorem for Discontinuous Functions
}

Citation for published version (APA):

Herings, P. J. J., van der Laan, G., Talman, A. J. J., \& Yang, Z. (2008). A Fixed Point Theorem for Discontinuous Functions. Operations Research Letters, 36, 89-93.

https://doi.org/10.1016/j.orl.2007.03.008

Document status and date:

Published: 01/01/2008

DOI:

10.1016/j.orl.2007.03.008

Document Version:

Publisher's PDF, also known as Version of record

Document license:

Taverne

Please check the document version of this publication:

- A submitted manuscript is the version of the article upon submission and before peer-review. There can be important differences between the submitted version and the official published version of record.

People interested in the research are advised to contact the author for the final version of the publication, or visit the DOI to the publisher's website.

- The final author version and the galley proof are versions of the publication after peer review.

- The final published version features the final layout of the paper including the volume, issue and page numbers.

Link to publication

\footnotetext{
General rights rights.

- You may freely distribute the URL identifying the publication in the public portal. please follow below link for the End User Agreement:

www.umlib.nl/taverne-license

Take down policy

If you believe that this document breaches copyright please contact us at:

repository@maastrichtuniversity.nl

providing details and we will investigate your claim.
}

Copyright and moral rights for the publications made accessible in the public portal are retained by the authors and/or other copyright owners and it is a condition of accessing publications that users recognise and abide by the legal requirements associated with these

- Users may download and print one copy of any publication from the public portal for the purpose of private study or research.

- You may not further distribute the material or use it for any profit-making activity or commercial gain

If the publication is distributed under the terms of Article $25 \mathrm{fa}$ of the Dutch Copyright Act, indicated by the "Taverne" license above, 


\title{
A fixed point theorem for discontinuous functions
}

\author{
P. Jean-Jacques Herings ${ }^{\mathrm{a}, *}{ }^{\mathrm{*}}$, Gerard van der Laan ${ }^{\mathrm{b}}$, Dolf Talman ${ }^{\mathrm{c}}$, Zaifu Yang ${ }^{\mathrm{d}}$ \\ ${ }^{a}$ Department of Economics, Maastricht University, P.O. Box 616, 6200 MD Maastricht, The Netherlands \\ ${ }^{\mathrm{b}}$ Department of Econometrics and Tinbergen Institute, Vrije Universiteit, De Boelelaan 1105, 1081 HV Amsterdam, The Netherlands \\ ${ }^{\mathrm{c}}$ Department of Econometrics \& Operations Research and CentER, Tilburg University, P.O. Box 90153, 5000 LE Tilburg, The Netherlands \\ ${ }^{\mathrm{d}}$ Faculty of Business Administration, Yokohama National University, Yokohama 240-8501, Japan
}

Received 20 April 2006; accepted 26 March 2007

Available online 4 July 2007

\begin{abstract}
Any function from a non-empty polytope into itself that is locally gross direction preserving is shown to have the fixed point property. Brouwer's fixed point theorem for continuous functions is a special case. We discuss the application of the result in the area of non-cooperative game theory.
\end{abstract}

(C) 2007 Elsevier B.V. All rights reserved.

MSC: 47H10; 65K10; 91A10

Keywords: Fixed point; Simplicial subdivision; Discontinuity; Discontinuous games

\section{Introduction}

Almost one century ago Brouwer proved a remarkable result saying that any continuous function from the $m$-dimensional unit ball to itself has a fixed point, a point that is mapped by the function into itself [3]. The Brouwer fixed point theorem was one of the early major achievements of algebraic topology. This celebrated theorem has been generalized in several ways. Well-known examples include Schauder, Borsuk-Ulam, and Kakutani $[13,2,9]$. For general surveys on the subject, we refer to $[14,8]$.

In this paper, we give a general condition, called the locally gross direction preserving property, under which a fixed point of a function $f$ from an arbitrary non-empty polytope to itself exists. The condition says that at any point $x$ which is not a fixed point of $f$ it must hold that the direction of $f(x)-x$ is grossly preserved in some neighborhood of $x$, in the sense that for any $y$ and $z$ in this neighborhood the inner product of $f(y)-y$ and $f(z)-z$ is non-negative. This novel condition

\footnotetext{
* Corresponding author.

E-mail addresses: p.herings@algec.unimaas.nl (P. Jean-Jacques Herings), glaan@feweb.vu.nl (G. van der Laan), talman@uvt.nl (D. Talman), yang@ynu.ac.jp (Z. Yang).
}

allows for various kinds of discontinuities and irregularities of the function $f$. On the other hand, any continuous function is locally gross direction preserving, so that Brouwer's fixed point theorem is covered by the result. It is worth pointing out that our approach is constructive.

Existence results of fixed points for discontinuous functions were previously given by Tarski and Caristi [16,4]. Our existence condition differs from Tarski's theorem in the sense that the latter theorem is restricted to a weakly increasing function on a (finite) sublattice. The monotonicity property is not required by our condition. Caristi's theorem concerns functions satisfying a non-expansion condition, which is also not required by our condition. It should be noted that a continuous function does not need to satisfy the conditions in Tarski's or Caristi's theorem and thus both these theorems do not cover Brouwer's theorem. We also demonstrate that a locally gross direction preserving function is not necessarily upper or lower semicontinuous. To the best of our knowledge, the new theorem seems to be the first that both allows discontinuities and covers Brouwer's theorem simultaneously in the single-valued case. From a practical viewpoint we would like to mention that discontinuities may arise naturally in various fields of study, such as engineering, economics and game theory; see e.g., [1,5,12]. 
In the latter paper, it is shown that better-reply secure games have pure strategy Nash equilibria. We will use our theorem to state some existence results for games with discontinuities, including the case of two-person non-zero-sum noisy games of timing.

This paper is organized as follows. Section 2 establishes the theorem and gives some examples to illustrate its generality. Section 3 presents some game theoretic applications.

\section{An existence theorem}

Let $P$ be a non-empty polytope in the $n$-dimensional Euclidean space $\mathbb{R}^{n}$ and let $f$ be a function mapping from $P$ to itself. We call $x^{*} \in P$ a fixed point of $f$ if $f\left(x^{*}\right)=x^{*}$, i.e., $f$ maps $x^{*}$ into itself. Brouwer proved that if $f$ is continuous on $P$, then $f$ has a fixed point.

In this paper, we provide a fixed point theorem that allows for discontinuities. More precisely, the existence of a fixed point is guaranteed when $f$ satisfies the so-called locally gross direction preserving property. A discrete version of this property was originally used in [17] to prove the existence of a fixed point in case the domain is a discrete set. For $x \in \mathbb{R}^{n}$ and $\delta>0$, let $B(x, \delta)$ denote the $n$-dimensional ball in $\mathbb{R}^{n}$ with center $x$ and radius $\delta$. Let $\mathbb{N}$ denote the set of natural numbers. For $h \in \mathbb{N}$, let $I_{h}$ denote the index set $\{1, \ldots, h\}$. For any $x, y \in \mathbb{R}^{n}, x^{\top} y$ stands for their inner product.

Definition 2.1. A function $f: P \rightarrow P$ is locally gross direction preserving if for every $x \in P$ for which $f(x) \neq x$, there exists $\delta>0$ such that for every $y, z \in B(x, \delta) \cap P$, the function $f$ satisfies

$(f(y)-y)^{\top}(f(z)-z) \geqslant 0$.

Now we are ready to present the main result of this paper. The proof is constructive and involves two basic arguments, one based on Brouwer's fixed point theorem and the other one, the use of locally gross direction preservingness, on basic algebra.

Theorem 2.2. Let $P$ be a non-empty polytope in $\mathbb{R}^{n}$ and let the function $f: P \rightarrow P$ satisfy the locally gross direction preserving property. Then $f$ has a fixed point.

Proof. Take a sequence of simplicial subdivisions, $\left(T_{k}\right)_{k \in \mathbb{N}}$, of $P$ with mesh size tending to zero if $k$ goes to infinity, i.e., for each $k \in \mathbb{N}, T_{k}$ is a finite collection of simplices whose union is $P$ and for which the intersection of any two simplices is either empty or a common face of both. Since $P$ is a polytope and therefore the convex hull of a finite number of points in $\mathbb{R}^{n}$, such a sequence exists; see for example [15]. For $k \in \mathbb{N}$, let $f^{k}$ denote the piecewise linear approximation of $f$ with respect to $T_{k}$, i.e., for $x \in P$,

$f^{k}(x)=\sum_{j=1}^{n+1} \lambda_{j} f\left(x^{j}\right)$ where $x^{1}, \ldots, x^{n+1}$ are the vertices of a simplex in $T_{k}$ containing $x$, and $\lambda_{1}, \ldots, \lambda_{n+1}$ are the unique non-negative numbers with sum equal to one satisfying

$x=\sum_{j=1}^{n+1} \lambda_{j} x^{j}$.

Since the function $f^{k}$ is piecewise linear and therefore continuous on $P$, the Brouwer fixed point theorem implies that for every $k \in \mathbb{N}$ there exists a fixed point $x^{k}$ of $f^{k}$.

Next we consider the sequence of points $\left(x^{k}\right)_{k \in \mathbb{N}}$. Since this sequence is a sequence of points in the compact set $P$, there exists a convergent subsequence. Without loss of generality we assume that the sequence $\left(x^{k}\right)_{k \in \mathbb{N}}$ itself converges to some $x^{*} \in P$. For $k \in \mathbb{N}$, let $\sigma^{k}$ be an $n$-dimensional simplex in $T_{k}$ with vertices $x^{k, 1}, \ldots, x^{k, n+1}$ containing $x^{k}$. Then there exist unique non-negative numbers $\lambda_{j}^{k}, j \in I_{n+1}$, with sum equal to 1 , satisfying

$x^{k}=\sum_{j=1}^{n+1} \lambda_{j}^{k} x^{k, j} \quad$ and $\quad f^{k}\left(x^{k}\right)=\sum_{j=1}^{n+1} \lambda_{j}^{k} f\left(x^{k, j}\right)$.

If $f\left(x^{*}\right)=x^{*}$, then $x^{*}$ is a fixed point of $f$ and the theorem has been proved. Suppose therefore that $x^{*}$ is not a fixed point of $f$. Then according to the condition of the theorem there exists $\delta^{*}>0$ such that for all $y, z \in B\left(x^{*}, \delta^{*}\right) \cap P$ it holds that

$(f(y)-y)^{\top}(f(z)-z) \geqslant 0$.

Since the sequence $\left(x^{k}\right)_{k \in \mathbb{N}}$ converges to $x^{*}$ and the mesh size of $T_{k}$ converges to zero when $k$ goes to infinity, we obtain that for every $j \in I_{n+1}$ the sequence $\left(x^{k, j}\right)_{k \in \mathbb{N}}$ converges to $x^{*}$. Hence, there exists $k^{*} \in \mathbb{N}$ such that for all $k \geqslant k^{*}$ it holds that $x^{k, j} \in B\left(x^{*}, \delta^{*}\right) \cap P$ for all $j \in I_{n+1}$ and therefore

$\left(f\left(x^{k, i}\right)-x^{k, i}\right)^{\top}\left(f\left(x^{k, j}\right)-x^{k, j}\right) \geqslant 0$,

for all $i, j \in I_{n+1}$. On the other hand, since $f^{k}\left(x^{k}\right)=x^{k}$, for all $k \in \mathbb{N}$, we have that

$\sum_{j=1}^{n+1} \lambda_{j}^{k}\left(f\left(x^{k, j}\right)-x^{k, j}\right)=0^{n}$,

where $0^{n}$ is the $n$-dimensional vector of zeros. Fix any $k \geqslant k^{*}$. Since $\sum_{j=1}^{n+1} \lambda_{j}^{k}=1$, there exists $j^{*} \in I_{n+1}$ satisfying $\lambda_{j^{*}}^{k}>0$. Premultiplying system (2) with $\left(f\left(x^{k, j^{*}}\right)-x^{k, j^{*}}\right)^{\top}$ we obtain $\sum_{j=1}^{n+1} \lambda_{j}^{k}\left(f\left(x^{k, j^{*}}\right)-x^{k, j^{*}}\right)^{\top}\left(f\left(x^{k, j}\right)-x^{k, j}\right)=0$.

Since, according to inequality (1), every term in this summation is non-negative, every term must be zero. So, taking $j=j^{*}$ we obtain that

$\left(f\left(x^{k, j^{*}}\right)-x^{k, j^{*}}\right)^{\top}\left(f\left(x^{k, j^{*}}\right)-x^{k, j^{*}}\right)=0$,

implying that $f\left(x^{k, j^{*}}\right)=x^{k, j^{*}}$. Hence $x^{k, j^{*}}$ is a fixed point of $f$. 
Observe that the proof distinguishes two possibilities. First the case where the limit point $x^{*}$ of the convergent sequence $\left(x^{k}\right)_{k \in \mathbb{N}}$ is a fixed point, and second, for $k$ large enough, any simplex in the converging sequence has at least one of its vertices as a fixed point. This also implies that the result does not follow from applying the Kakutani fixed point theorem to the mapping $F$ defined as the convex closure of $f$. By definition of the convex closure, $F$ is upper semi-continuous and thus there exists a point $x^{*}$ satisfying $x^{*} \in F\left(x^{*}\right)$. However this procedure does not guarantee that a fixed point of $F$ is also a fixed point of $f$. Therefore, the proof of Theorem 2.2 does not follow as an application of the Kakutani fixed point theorem. This can be also understood by observing that the Kakutani fixed point theorem generalizes the Brouwer fixed point theorem to correspondences, but it is identical to Brouwer's theorem when the correspondence is single-valued. In contrast, Theorem 2.2 generalizes Brouwer's fixed point theorem to discontinuous singlevalued functions. The following lemma shows that Brouwer's theorem is indeed a special case of Theorem 2.2, in the sense that a continuous function satisfies the locally gross preserving condition.

Corollary 2.3. Let $P$ be a non-empty polytope in $\mathbb{R}^{n}$ and let $f: P \rightarrow P$ be a continuous function. Then $f$ is locally gross direction preserving and therefore has a fixed point.

Proof. Take any point $x$ in $P$ and suppose that $f(x) \neq x$. Clearly, $(f(x)-x)^{\top}(f(x)-x)>0$. Consider the function $g$ : $P \times P \rightarrow \mathbb{R}$ defined by

$g(y, z)=(f(y)-y)^{\top}(f(z)-z)$.

Since the function $f$ is continuous, the function $g$ is also continuous. So, since $g(x, x)>0$, there exists $\delta>0$ so that for all $y, z \in B(x, \delta) \cap P$ it holds that $g(y, z)>0$. Hence $f$ is locally gross direction preserving and the result follows from Theorem 2.2.

Corollary 2.3 shows that the locally gross direction preserving condition indeed gives a natural and novel relaxation of continuity. Although the condition allows for various kinds of discontinuities, the condition puts sufficient properties on the function to guarantee the existence of a fixed point. To illustrate and clarify this, we consider the next example of a onedimensional function on [0,2], where at any $x, f^{-}(x)$ denotes the lower limit of $f$ from the left and $f^{+}(x)$ the upper limit of $f$ from the right.

Example 2.4. Let $f:[0,2] \rightarrow[0,2]$ be continuous at any $x \in$ $[0,2]$, except at $x=1$. Without loss of generality we assume that $f(x)>x$ for all $x<1$ (otherwise there is a fixed point $x^{*}$ satisfying $\left.x^{*}<1\right)$. So, $f^{-}(1) \geqslant 1$. Now the locally gross direction preserving condition requires that $f(1) \geqslant 1$, since $f(1)<1$ contradicts that for every $y$ and $z$ in some $B(1, \delta)$ it holds that $(f(y)-y)^{\top}(f(z)-z) \geqslant 0$. Further, when $f^{+}(1)>1$, then there exists a fixed point $x^{*}>1$. So, suppose $f^{+}(1) \leqslant 1$. Then the locally gross direction preserving property requires that either (i) $f(1)=1$ and thus $x^{*}=1$ is a fixed point, or (ii) $f(1)>1$ and there exists some $y, 1<y \leqslant 2$, such that $f(x) \geqslant x$ for all $1<x \leqslant y$. In the latter case there is a fixed point $x^{*}>1$. In particular it may occur that $f(x)=x$ for all $1<x \leqslant y$, corresponding to the case in the proof that for $k$ large enough any simplex in the converging sequence has at least one of it vertices as a fixed point. Observe that the locally gross direction preserving property excludes that $f(1)>1$ and $f^{+}(1)<1$ and also that $f(1)<1$ and $f^{-}(1)>1$.

More generally, the locally gross direction preserving condition requires that if $x$ is not a fixed point of $f$, there exists a neighborhood of $x$ such that for any two points $y$ and $z$ in this neighborhood it holds that the vectors $f(y)-y$ and $f(z)-z$ either make a sharp angle with each other or are orthogonal to each other, i.e., the direction of these two vectors is grossly preserved. This condition replaces continuity at $x$. If $x$ is a fixed point of $f$ nothing about $f$ around $x$ is required.

Locally gross direction preserving does not require that the function is monotone non-decreasing, a property required by Tarski's theorem. On the other hand, a function satisfying Tarski's theorem does not need to be locally gross direction preserving. For example, if $f(x)=\frac{1}{3}(x+1)$ for $0 \leqslant x \leqslant 1$ and $f(x)=\frac{1}{3}(x+3)$ for $1<x \leqslant 2$, then $f$ is monotone increasing on the interval $[0,2]$, but $f$ is not locally gross direction preserving at $x=1$.

It should also be noticed that a locally gross direction preserving function may be neither lower nor upper semi-continuous. A function $f:[0,1] \rightarrow[0,1]$ is said to be lower semi-continuous if for all sequences $\left(x^{k}\right)_{k \in \mathbb{N}}$ with $x^{k} \in[0,1]$ for all $k \in \mathbb{N}$, such that $x^{k} \rightarrow \bar{x}$ it holds that $\lim _{\inf _{k \rightarrow \infty}} f\left(x^{k}\right) \geqslant f(\bar{x})$. A function $f:[0,1] \rightarrow[0,1]$ is said to be upper semi-continuous if for all sequences $\left(x^{k}\right)_{k \in \mathbb{N}}$ with $x^{k} \in[0,1]$ for all $k \in \mathbb{N}$, such that $x^{k} \rightarrow \bar{x}$ it holds that $\lim _{\sup _{k \rightarrow \infty}} f\left(x^{k}\right) \leqslant f(\bar{x})$. Clearly, if $f(\bar{x})>\lim \inf _{k \rightarrow \infty} f\left(x^{k}\right)>\bar{x}$, then $f$ is locally gross direction preserving at $\bar{x}$, but $f$ is not lower semi-continuous at $\bar{x}$. Similarly, if $f(\bar{x})<\lim \sup _{k \rightarrow \infty} f\left(x^{k}\right)<\bar{x}$, then $f$ is locally gross direction preserving at $\bar{x}$, but $f$ is not upper semicontinuous at $\bar{x}$.

Finally, we shall extend the theorem to correspondences $\varphi$ on a non-empty polytope $P$. We call the function $f: P \rightarrow P$ a selection of $\varphi$ if $f(x) \in \varphi(x)$ for every $x \in P$, and $x^{*} \in P$ a fixed point of $\varphi$ if $x^{*} \in \varphi\left(x^{*}\right)$.

Theorem 2.5. Let $P$ be a non-empty polytope in $\mathbb{R}^{n}$ and let $\varphi: P \rightarrow P$ be a correspondence. If there exists a selection $f$ of $\varphi$ such that for every $x \in P$ for which $x \notin \varphi(x)$, there exists $\delta>0$ such that for every $y, z \in B(x, \delta) \cap P$, the selection $f$ satisfies

$(f(y)-y)^{\top}(f(z)-z) \geqslant 0$,

then $\varphi$ has a fixed point.

Proof. Let $f$ be a selection of $\varphi$ satisfying the conditions of the theorem. Consider the selection $g$ of $\varphi$ which is such that $g(x)=f(x)$ for every $x \in P$ for which $x \notin \varphi(x)$, and $g(x)=x$, otherwise. Let $x \in P$ be such that $x \notin \varphi(x)$ and let $\delta>0$ be 
such that for every $y, z \in B(x, \delta) \cap P$,

$(f(y)-y)^{\top}(f(z)-z) \geqslant 0$.

Then also, for every $y, z \in B(x, \delta) \cap P$,

$(g(y)-y)^{\top}(g(z)-z) \geqslant 0$.

Indeed, if $y \in \varphi(y)$ or $z \in \varphi(z)$, then it holds that $(g(y)-$ $y)^{\top}(g(z)-z)=0$. If $y \notin \varphi(y)$ and $z \notin \varphi(z)$, then we obtain

$(g(y)-y)^{\top}(g(z)-z)=(f(y)-y)^{\top}(f(z)-z) \geqslant 0$.

The function $g$ satisfies the assumptions of Theorem 2.2, so has a fixed point. This point is also a fixed point of $\varphi$.

Observe that this theorem does not require the condition in the Kakutani theorem that $\varphi(x)$ is convex for every $x \in P$. In addition, this new theorem is also a strict and meaningful generalization of Theorem 2.2. We can illustrate this point by the following simple example. A correspondence is defined by $\varphi(x)=\{1\}$ for $1 \leqslant x<\frac{1}{2}, \varphi(x)=\left\{\frac{1}{2}, 1\right\}$ for $x=\frac{1}{2}$, and $\varphi(x)=\{0\}$ for $\frac{1}{2}<x \leqslant 1$. Define a function by $f(x)=1$ for $1 \leqslant x \leqslant \frac{1}{2}$ and $f(x)=0$ for $\frac{1}{2}<x \leqslant 1$. So $f$ is a selection of $\varphi$ and moreover $f$ satisfies the condition of Theorem 2.5. So $\varphi$ has a fixed point. In fact, $\frac{1}{2}$ is a fixed point of $\varphi$. Nevertheless, the function $f$ does not have a fixed point at all.

\section{Nash equilibrium in discontinuous games}

Discontinuities arise naturally in various disciplines, such as economics and game theory; see, e.g., [1,5,12]. In this section, we give some results for the existence of a Nash equilibrium in discontinuous games. A non-cooperative game consists of a finite number of players, say, $N$. Player $i, i \in I_{N}$, has available a set of actions, denoted by $S^{i}$. For any $i \in I_{N}$, the set $S^{i}$ is an $m_{i}$-dimensional polytope in $\mathbb{R}^{n_{i}}$ for some non-negative integers $m_{i}$ and $n_{i}$. The cartesian product $\prod_{i=1}^{N} S^{i}$ is called the strategy space of the game and is denoted by $S$. An element $x=$ $\left(x_{1}, \ldots, x_{N}\right)$ of $S$ is called a strategy combination. A strategy combination $x \in S$ yields a payoff $u_{i}\left(x_{i}, x_{-i}\right)$ to player $i$, where $x_{-i}=\left(x_{j}\right)_{j \neq i}$ denotes the actions of players other than player $i$ in strategy $x$. A strategy combination $x^{*} \in S$ is a Nash equilibrium if for every player $i \in I_{N}$ action $x_{i}^{*}$ maximizes the payoff of player $i$ given that the other players choose $x_{-i}^{*}$, i.e., for all $i \in I_{N}$

$u_{i}\left(x_{i}^{*}, x_{-i}^{*}\right) \geqslant u_{i}\left(x_{i}, x_{-i}^{*}\right)$ for all $x_{i} \in S^{i}$.

For $x_{-i} \in \prod_{j \neq i} S^{j}$, let $b_{i}\left(x_{-i}\right)$ be a best reply of player $i$ when the other players choose actions according to $x_{-i}$, i.e., $b_{i}\left(x_{-i}\right)$ maximizes $u_{i}\left(x_{i}, x_{-i}\right)$ over $x_{i} \in S^{i}, i \in I_{N}$. Clearly, $x^{*} \in S$ is a Nash equilibrium if $b_{i}\left(x_{-i}^{*}\right)=x_{i}^{*}$ for all $i \in I_{N}$. It follows immediately from the Brouwer fixed point theorem that if every best reply function $b_{i}, i \in I_{N}$, is continuous, then there exists a Nash equilibrium. Here we show that a Nash equilibrium exists under the following condition on the best reply functions, which is weaker than continuity.
Theorem 3.1. Let $S=\prod_{i=1}^{N} S^{i}$ be the strategy space of a noncooperative game, the cartesian product of $N$ non-empty polytopes and let $b_{i}: \prod_{j \neq i} S^{j} \rightarrow S^{i}$, for $i \in I_{N}$, be best reply functions satisfying that for every $x \in S$ for which $b_{j}\left(x_{-j}\right) \neq$ $x_{j}$ for some $j \in I_{N}$ there exists $\delta>0$ such that for every $y$, $z \in B(x, \delta) \cap S$ it holds that

$\sum_{i=1}^{N}\left(b_{i}\left(y_{-i}\right)-y_{i}\right)^{\top}\left(b_{i}\left(z_{-i}\right)-z_{i}\right) \geqslant 0$.

Then there exists a Nash equilibrium.

Proof. We define the function $r_{i}$ from $S$ to $\mathbb{R}^{n_{i}}$ by $r_{i}(x)=$ $b_{i}\left(x_{-i}\right)$. The condition in the theorem implies that for all $x \in S$ for which $b_{j}\left(x_{-j}\right) \neq x_{j}$ for some $j \in I_{N}$ there exists $\delta>0$ satisfying that for every $y, z \in B(x, \delta) \cap S$ we have

$\sum_{i=1}^{N}\left(r_{i}(y)-y_{i}\right)^{\top}\left(r_{i}(z)-z_{i}\right) \geqslant 0$.

For $x \in S$, define $r(x)=\left(r_{1}(x), \ldots, r_{N}(x)\right)$. Clearly, the set $S$ is an $m$-dimensional polytope in $\mathbb{R}^{n}$, with $m=\sum_{j=1}^{N} m_{j}$ and $n=\sum_{j=1}^{N} n_{j}$. Then $r$ is a function from $S$ into itself and for every $x \in S$ for which $r(x) \neq x$ there exists $\delta>0$ satisfying that for every $y, z \in B(x, \delta) \cap S$ we have

$(r(y)-y)^{\top}(r(z)-z) \geqslant 0$,

i.e., the function $r$ satisfies the conditions of Theorem 2.2 with $P$ equal to $S$. Hence, the function $r$ has a fixed point $x^{*}$ on $S$, i.e., $r\left(x^{*}\right)=x^{*}$. Clearly, a fixed point $x^{*}$ of $r$ satisfies $b_{i}\left(x_{-i}^{*}\right)=x_{i}^{*}$ for all $i \in I_{N}$.

Two remarks are in order. First, using the argument of Corollary 2.3 , it is easy to show that if all functions $b_{i}$ are continuous, the conditions in Theorem 3.1 are automatically satisfied. Second, by applying Theorem 2.5 , we can easily extend Theorem 3.1 to allow for best response correspondences instead of best reply functions.

In the rest of this section, we discuss a specific class of discontinuous games. In [12] games with discontinuous payoff functions are studied. For such games best reply functions or correspondences may not be well defined and so the existence of a Nash equilibrium cannot be derived from the existence of a fixed point of the best replies. Reny introduces the class of socalled better-reply secure games and shows that such a game has a pure strategy Nash equilibrium. Any game with continuous payoff functions is better-reply secure, but the class also allows for discontinuities. For instance, as in [12], we consider the class of two-person, non-zero-sum, noisy games of timing on the unit square, which can be traced back to [10]. The payoff to player $i=1,2$ is given by

$u_{i}\left(x_{i}, x_{-i}\right)= \begin{cases}\ell_{i}\left(x_{i}\right) & \text { if } x_{i}<x_{-i}, \\ \phi_{i}\left(x_{i}\right) & \text { if } x_{i}=x_{-i}, \\ m_{i}\left(x_{-i}\right) & \text { if } x_{i}>x_{-i},\end{cases}$

where (i) both $\ell_{i}$ and $m_{i}$ are continuous on $[0,1]$ and $\ell_{i}$ is nondecreasing on $[0,1]$ and (ii) for every $x \in[0,1]$ it holds 
that $\phi_{i}(x) \in \operatorname{co}\left\{\ell_{i}(x), m_{i}(x)\right\}$, the convex hull of $\ell_{i}(x)$ and $m_{i}(x)$, and $\operatorname{sgn}\left(\ell_{i}(x)-\phi_{i}(x)\right)=\operatorname{sgn}\left(\phi_{-i}(x)-m_{-i}(x)\right)$ with the sign function $\operatorname{sgn}(y)=-1$ for $y<0, \operatorname{sgn}(y)=0$ for $y=0$, and $\operatorname{sgn}(y)=1$ for $y>0$. For this class of games, [11] presents necessary and sufficient conditions for the existence of a mixed strategy equilibrium, while [12] shows that every such game is better-reply secure and therefore possesses a Nash equilibrium in pure strategies.

Applying Theorem 2.2 we will establish the existence of a symmetric Nash equilibrium in pure strategies. Notice that since the payoff functions are not continuous, the best replies are not defined everywhere. Instead we define the function $\beta:[0,1] \rightarrow$ $[0,1]$ given by

$\beta(x)= \begin{cases}1 & \text { if } \ell_{1}(x)<m_{1}(x), \\ x & \text { if } \ell_{1}(x)=m_{1}(x), \\ 0 & \text { if } \ell_{1}(x)>m_{1}(x) .\end{cases}$

We show that $\beta$ is locally gross direction preserving on $[0,1]$, which establishes the existence of a fixed point. Then we prove that a fixed point of $\beta$ induces a symmetric Nash equilibrium in pure strategies. See [7] or [6] for other approaches to show the existence of a Nash equilibrium by using a fixed point function not being a best reply function.

First, for every $x$ at which $\ell_{1}(x)<m_{1}(x)$ or $\ell_{1}(x)>m_{1}(x), \beta$ is continuous and so certainly locally gross direction preserving. For every $x$ at which $\ell_{1}(x)=m_{1}(x), x$ is a fixed point of $\beta$. As a result, $\beta$ meets the condition of Theorem 2.2 and thus has a fixed point on $[0,1]$.

Next we show that a fixed point $x$ of $\beta$ induces the symmetric Nash equilibrium in pure strategies $(x, x)$. We need to consider three cases.

1. If $x=0$, then $\ell_{1}(0) \geqslant \phi_{1}(0) \geqslant m_{1}(0)$, and, using the sign condition, $\ell_{2}(0) \geqslant \phi_{2}(0) \geqslant m_{2}(0)$, so

$u_{1}(0,0)=\phi_{1}(0) \geqslant m_{1}(0)=u_{1}\left(x_{1}, 0\right)$ for every $x_{1}>0$,

$u_{2}(0,0)=\phi_{2}(0) \geqslant m_{2}(0)=u_{2}\left(0, x_{2}\right)$ for every $x_{2}>0$.

Hence $(x, x)$ is a Nash equilibrium.

2. If $0<x<1$, then $\ell_{1}(x)=\phi_{1}(x)=m_{1}(x)$, and by the sign condition $\ell_{2}(x)=\phi_{2}(x)=m_{2}(x)$, so for $x_{1}<x$,

$u_{1}(x, x)=\phi_{1}(x) \geqslant \ell_{1}\left(x_{1}\right)=u_{1}\left(x_{1}, x\right)$,

where the inequality uses the fact that $\ell_{1}$ is non-decreasing, and for $x_{1}>x$,

$u_{1}(x, x)=\phi_{1}(x)=m_{1}(x)=u_{1}\left(x_{1}, x\right)$.

A symmetric argument shows that player 2 does not have a profitable deviation and thus again $(x, x)$ is a Nash equilibrium.
3. If $x=1$, then $\ell_{1}(1) \leqslant \phi_{1}(1) \leqslant m_{1}(1)$, so $\ell_{2}(1) \leqslant \phi_{2}(1) \leqslant$ $m_{2}(1)$, and

$u_{1}(1,1)=\phi_{1}(1) \geqslant \ell_{1}\left(x_{1}\right)=u_{1}\left(x_{1}, 1\right)$ for every $x_{1}<1$,

$u_{2}(1,1)=\phi_{2}(1) \geqslant \ell_{2}\left(x_{2}\right)=u_{2}\left(1, x_{2}\right)$ for every $x_{2}<1$.

Again, $(x, x)$ is a Nash equilibrium.

\section{Acknowledgments}

This research was carried out while Jean-Jacques Herings, Gerard van der Laan and Zaifu Yang were visiting the CentER for Economic Research, Tilburg University, during the summer of 2004. Jean-Jacques Herings would like to thank the Netherlands Organisation for Scientific Research (NWO) for financial support. Zaifu Yang gratefully acknowledges financial support by CentER and the Netherlands Organisation for Scientific Research (NWO). The authors would like to thank an anonymous referee for helpful suggestions and comments.

\section{References}

[1] K.J. Arrow, F.H. Hahn, General Competitive Analysis, Holden-Day, San Francisco, 1971.

[2] K. Borsuk, Drei Sätze über die n-dimensionale euklidische Sphäre, Fund. Math. 20 (1933) 177-190.

[3] L.E.J. Brouwer, Über Abbildung von Mannigfaltigkeiten, Math. Ann. 71 (1912) 97-115.

[4] J. Caristi, Fixed point theorems for mappings satisfying inwardness conditions, Trans. Am. Math. Soc. 215 (1976) 241-251.

[5] D. Fudenberg, J. Tirole, Game Theory, MIT Press, Boston, 1991.

[6] J.D. Geneakoplos, Nash and Walras equilibrium via Brouwer, Econ. Theory 21 (2003) 585-603.

[7] P.J.J. Herings, Two simple proofs of feasibility of the linear tracing procedure, Econ. Theory 15 (2000) 485-490.

[8] V.I. Istratescu, Fixed Point Theory: An Introduction, Reidel Publishing, Dordrecht, 1981.

[9] S. Kakutani, A generalization of Brouwer's fixed point theorem, Duke Math. J. 8 (1941) 457-459.

[10] S. Karlin, Mathematical Models and Theory in Games, Programming and Economics, Addison-Wesley, Reading, MA, 1959.

[11] C. Pitchik, Equilibria of a two-person non-zero-sum noisy game of timing, Int. J. Game Theory 10 (1982) 207-221.

[12] P.J. Reny, On the existence of pure and mixed strategy Nash equilibria in discontinuous games, Econometrica 67 (1999) 1029-1056.

[13] J. Schauder, Der Fixpunktsatz in Funktionalraümen, Stud. Math. 2 (1930) 171-180.

[14] R. Smart, Fixed Point Theorems, Cambridge University Press, Cambridge, 1974.

[15] A.J.J. Talman, Y. Yamamoto, A simplicial algorithm for stationary point problems on polytopes, Math. Oper. Res. 14 (1989) 383-399.

[16] A. Tarski, A lattice-theoretical fixed point theorem and its applications, Pac. J. Math. 5 (1955) 285-309.

[17] Z. Yang, Discrete fixed point analysis and its applications, FBA Discussion Paper 210, Yokohama National University, Yokohama, 2004. 